\title{
Adapting clinical practice guidelines to local context and assessing barriers to their use
}

\author{
Margaret B. Harrison RN PhD, France Légaré MD PhD, Ian D. Graham PhD, Béatrice Fervers MD PhD
}

$\mathrm{T}$ he knowledge-to-action cycle represents a framework for the implementation of knowledge. ${ }^{1}$ As discussed in the first article in this series, the action phases of this cycle were derived from a review of 31 theories of planned action. ${ }^{2}$ Included in this cycle (Figure 1) are the processes needed to implement knowledge in health care settings. In this paper, we address the adaptation of the knowledge to the local context and assessment of barriers to and facilitators of the use of knowledge. The action cycle is a dynamic and iterative process with each phase informing the others.

\section{Why adapt guidelines for local use?}

Using the best evidence is a fundamental aspect of quality health care and valid guidelines for clinical practice are an important tool to inform evidence-based practices. Guidelines are derived from synthesized evidence that has been translated into specific practice-oriented recommendations. ${ }^{3}$ The production of guidelines has been promoted and supported by governments and professional organizations as a mechanism for reducing variations in practice. Many countries have infrastructure at the national or regional level dedicated to synthesizing evidence and producing guidelines, as well as incentives designed to support practices guided by current recommendations of guidelines. ${ }^{4}$

The goals of these initiatives differ depending on the political context and the health care system. For instance, in the United Kingdom, the National Health Service has infrastructure and incentives to deliver care guided by recommendations of guidelines. National bodies such as the National Institute for Health and Clinical Excellence ${ }^{5}$ are dedicated to synthesizing evidence and producing guidelines for use within the National Health Service. To assess the uptake and adherence to guideline-based care, auditing functions exist across trusts (i.e., regions) in the National Health Service. Despite these efforts, evaluation of these strategies for implementation show that overall conformity of practices lags behind expectations. ${ }^{6}$

Although high-quality guidelines may be seen as necessary, they are not sufficient to ensure evidence-based decisionmaking. Uptake of knowledge does not occur with simple dissemination and usually requires a substantive, proactive effort to encourage use at the point of decision-making. The gap

\section{Key points}

- Clinical practice guidelines can be adapted to local circumstances and settings to avoid duplication of efforts and optimize use of resources.

- The ADAPTE process is an approach to adapting guidelines to local contexts through the explicit participation of relevant decision-makers.

- Assessing barriers to and facilitators of the use of knowledge is closely linked to the adaptation and uptake of the evidence.

between valid recommendations of guidelines and delivery of care based on this evidence may be due to numerous barriers. For instance, clinicians may not have the requisite skills and expertise to implement a recommended action (e.g., being unfamiliar with how to initiate or titrate a new medication), or the setting may not have the mandatory equipment or its staff the time to deliver a guideline's recommendation. ${ }^{3}$

Although guidelines provide evidence in a more usable form for clinicians than a plethora of primary studies, an important and additional necessary step is the adaptation of the guideline to the local context of use. National and international bodies have made major efforts to improve the quality and rigour of guidelines, ${ }^{8,9}$ but less investment has been made in understanding how guidelines can be targeted to the local context of health care. By local, we mean a continuum of contexts that could range from a single clinic to a hospital, region or nation.

Customizing a clinical practice guideline to a particular organization may improve acceptance and adherence. Active involvement of the end-users of the guideline in this process has been shown to lead to significant changes in practice. ${ }^{10-13}$ For example, local and regional adaptations of international

From the School of Nursing, the Department of Community Health and Epidemiology and the Practice and Research in Nursing Group (Harrison), Queen's University, Kingston, Ont.; the Department of Family Medicine and Emergency Medicine, Université Laval, and Centre de Recherche du Centre Hospitalier Universitaire de Québec (Légaré), Québec, Que.; the Canadian Institutes of Health Research and the School of Nursing (Graham), Department of Epidemiology and Community Medicine, University of Ottawa, Ottawa, Ont.; and the Oncology Guideline Programme (Fervers), French Federation of Cancer Centers, Centre Léon-Bérard and Université Lyon, Lyon, France.

Cite as CMAJ 2009.DOI:10.1503/cmaj.081232 
evidence-based practice guidelines have become mandatory for the care of patients with cancer in France. ${ }^{14}$ For many regions and territorial jurisdictions, de novo development of guidelines is not feasible because of lack of time, expertise and resources, and thus taking advantage of existing highquality guidelines is sensible. ${ }^{15-17}$

Adaptation of existing high-quality guidelines for local use is an approach with the potential to reduce duplication of effort and enhance applicability. National guidelines often lack details on applicability and description of the changes in the organization of care required to apply the recommendations..$^{18}$ Adaptation of evidence may promote local uptake of evidence through a sense of ownership by the end-users who are engaged in this process. However, customizing a guideline to local conditions could weaken the integrity of the evidence base. We outline a systematic, participatory approach for evaluating and adapting available guidelines to a local context of use while maintaining the quality and validity of the guideline. Whether evidence is provided in the format of syntheses of knowledge, patient decision aids or clinical practice guidelines, end-users must consider if or how it could be adapted to the local context and the same principles can be applied to ensure these factors are considered before implementation of the evidence.

To illustrate how to adapt guidelines, we will use a recent study that was performed to improve community care of individuals living with venous ulcers of the leg. ${ }^{10,13}$ Regional managers of home care were concerned about costs of supplies,

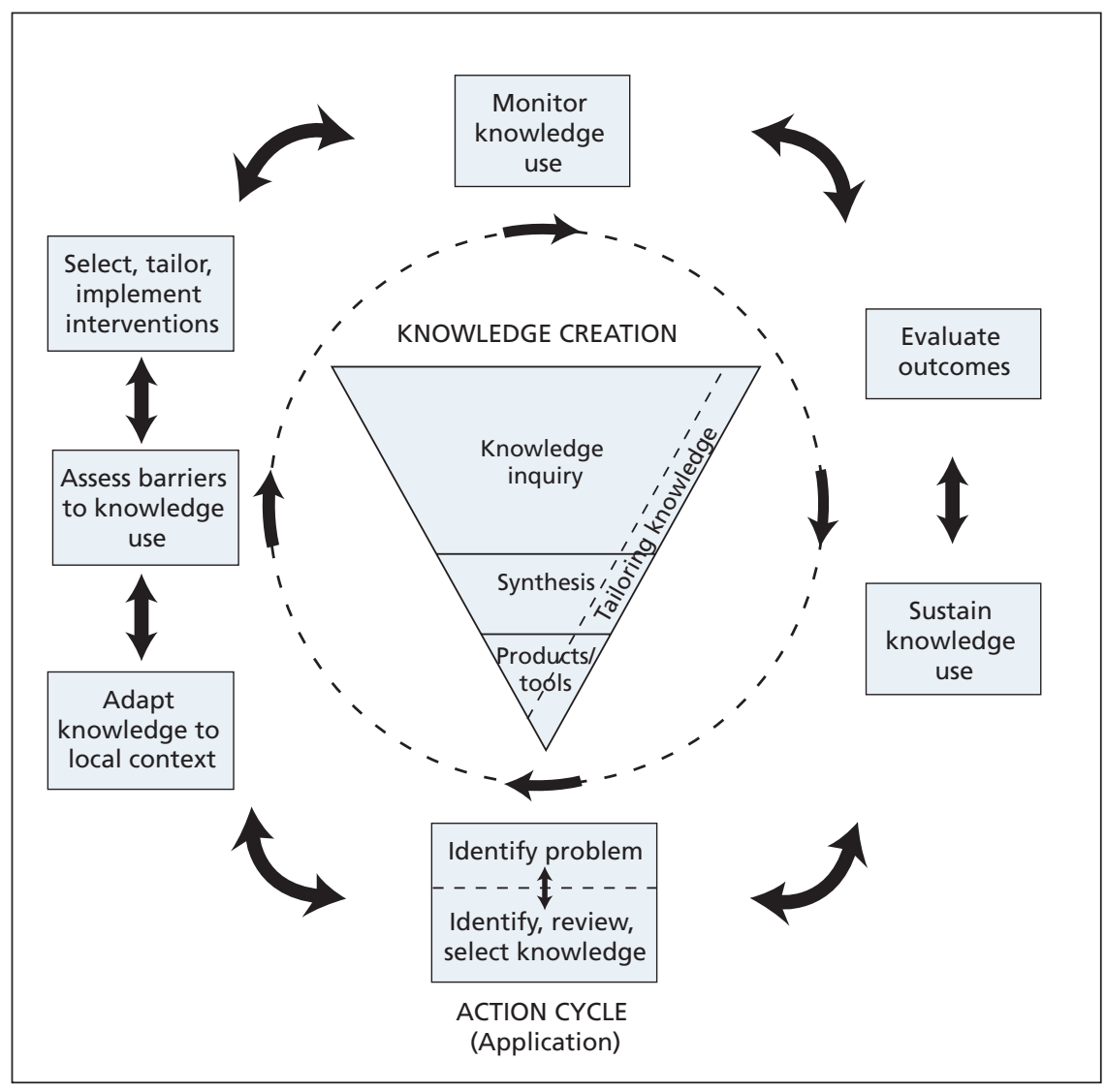

Figure 1: The knowledge-to-action cycle. amount of nursing time, and frequency of visiting for clients with ulcers of the leg. A regional task force was developed to review existing practice guidelines to help guide the care plan. The task force identified that many of these guidelines were from international bodies and would require adaptation to the local context.

\section{How are guidelines adapted for local use?}

Existing guidelines can be evaluated and customized to fit local circumstances through an active, systematic and participatory process. This process must preserve the integrity of the evidence-based recommendations when differences in organizational, regional or cultural circumstances may legitimately require important variations in recommendations..$^{8,914-16,19} \mathrm{In}$ adapting a guideline, consideration is given to local evidence, such as specific health questions relevant to a local context of use; to specific needs, priorities, legislation, policies and resources; to scopes of practice within the local health services; and to fit within existing models of delivery in the targeted setting. Adapting the guideline to this local evidence is assumed to improve uptake of the guidelines.

Returning to our example involving care of ulcers of the leg, the task force collectively assessed the quality of individual guidelines and their recommendations. They developed a protocol that was feasible to implement locally and that was endorsed by stakeholders. The guideline was condensed to a one-page algorithm to enhance use by the clinicians, and documentation forms were created for collection of clinical data. For example, to streamline the process of assessment and facilitate application of evidence-based care, documentation forms were created to collect information about the cause of the ulcer, with venous symptoms and history on one side of the page and arterial symptoms on the other.

With the exception of a few studies such as this collaboration for care of ulcers of the leg, ${ }^{10,13,17}$ no validated process for the adaptation of guidelines has been documented. Recently, the Canadian work ${ }^{16}$ in this area was integrated with an international initiative known as the ADAPTE collaboration (www.adapte.org). ${ }^{19}$ This collaboration is a group of researchers and developers, implementers and users of guidelines whose aim is to enhance the use of research-based evidence through more efficient development and implementation of practice guidelines. The ADAPTE process was developed to facilitate the creation of efficient, high-quality adapted guidelines. The process engages end-users in the guideline adaptation process to address specific health-related questions relevant to its context of use. The goal is to establish a standard of being transparent, 
rigorous and replicable based on the following core principles:

- Respect of evidence-based principles in the development of guidelines, ${ }^{20}$

- Use of reliable and consistent methods to ensure the quality of the adapted guideline, ${ }^{9,19}$

- Participation of key stakeholders to foster acceptance and ownership of the adapted guideline and ultimately promote its use, ${ }^{13,14}$

- Consideration of context during adaptation to ensure relevance for local practice and policy, ${ }^{13,21}$

- Transparent reporting to promote confidence in the recommendations of the adapted guideline,, 920

- Use of a flexible format to accommodate specific needs and circumstances, ${ }^{18,22}$ and

- Respect for and acknowledgement of guideline materials used as sources.

\section{What is the ADAPTE process?}

The ADAPTE process consists of three main phases, including planning and set-up, adaptation, and development of a final product (Box 1). The set-up phase outlines the necessary tasks to be completed before the process of adaptation, including identifying necessary skills and resources, and designing the panel. The panel should include relevant end-users of the guideline, such as clinicians, managers and patients.

The phase of adaptation assists in moving from selection of a topic to identification of specific clinical questions; in searching for, retrieving and assessing guidelines; in decisionmaking around adaptation; and in preparing the draft version of the adapted guideline. Assessment of the retrieved guidelines involves evaluation of their quality (i.e., using the AGREE [Appraisal of Guidelines Research and Evaluation] instrument ${ }^{9,23}$ ), currency (i.e., how up-to-date they are) and consistency (i.e., congruence of the recommendation with the underlying evidence). Assessment also consists of the examination of the acceptability (i.e., to clinicians and patients) and applicability (i.e., feasibility of applying recommendations) of the guidelines' recommendations within the proposed context of use. This evaluation provides an explicit basis for informed and transparent decision-making around the selection and modification of guidelines used as sources. This process can result in different alternatives ranging from adopting a guideline unchanged, to translation of language and adaptation of format, to modification and updating of single recommendations, to the production of a customized guideline based on various guidelines used as sources. The finalization phase includes external review, feedback from relevant stakeholders, and consultation with the developers of source guidelines. Establishing a process for updating the adapted guideline and writing the final document are the last stages.

The ADAPTE process is supported by tools on the ADAPTE website, including a manual and toolkit. For each module, the manual provides a detailed description of the aims and tasks, the products and deliverables, and the skills and organizational requirements necessary to undertake the tasks. An example related to the adaptation of guidelines for screening for cervical cancer is provided throughout the modules. In the toolkit, 19 tools or instruments are offered to help structure the process and collect necessary information for decisionmaking.

For example, tool number 2 offers a comprehensive search strategy to help in identifying existing guidelines by searching websites of sources of guidelines (e.g., guideline-related clearinghouses, known developers' sites, specialty organizations) and MEDLINE. Tool number 6 helps a group convert the topic of the guideline into a set of clear and focused key questions before the process of adaptation. Tool number 15 proposes a series of structured questions and criteria to guide the assessment of and discussion on whether a recommendation of a guideline is applicable or acceptable in the planned context of use and to identify the organizational changes that may be needed to deliver the recommendation. Steps and tools are flexible and have been designed to allow for alteration in the sequence in which they are used to fit with users' time or restraints in resources.

Adapting a guideline or other tool of knowledge is a key component of the knowledge-to-action cycle. The adaptation process also integrates other steps of the cycle, including assessment of barriers to and facilitators of use of knowledge, which is necessary both for adapting and implementing the guideline.

\section{Key concepts for assessment}

The use of a framework is important for assessing barriers because it helps researchers and practitioners identify research questions, generate testable hypotheses, assess outcomes using valid and reliable instruments and make valid inferences from their results. A framework would ensure that researchers can elaborate theory-based interventions that have the potential for increasingly effective implementation of knowledge into clinical practice. ${ }^{24}$ More importantly, the use of a framework also provides the foundation for the tools that help busy clinicians implement practice guidelines.

One of the more often-cited conceptual frameworks regarding barriers to use of knowledge in health care is the Clinical Practice Guidelines Framework for Improvement by Cabana and colleagues. ${ }^{25}$ This framework was based on an extensive search of the literature for barriers to adherence by physicians to clinical practice guidelines and was organized according to knowledge, attitudes and behaviour of physicians. ${ }^{26}$ Of 5658 potentially eligible articles, Cabana and colleagues identified 76 published studies describing at least one barrier to adherence to clinical practice guidelines. The included articles reported on a total of 293 potential barriers to adherence to guidelines by physicians. These barriers included unawareness of the existence of the guideline $(n=46)$, unfamiliarity with the recommendations of guidelines $(n=31)$, disagreement with the recommendations $(n=33)$, lack of self-efficacy (i.e., feeling one is unable to carry out the recommendations) $(n=19)$, outcome expectancy (i.e., the perception that health outcomes will be changed if the recommendations are followed) $(n=8)$, inability to overcome the inertia of previous practice $(n=14)$ and presence of external barriers to following the recommendations $(n=34) .^{25}$

Espeland and Baerheim ${ }^{27}$ proposed a revised and extended classification of barriers based on the Clinical Practice Guide- 
Box 1: Phases, modules and steps in the adaptation of a guideline using the ADAPTE process. A scenario of care of patients with leg ulcers is included as an example. (Part 1 of 2)

PHASE I - Set-up

Module: Preparation

1. Establish an organizing committee, working panel or resource team.

This group will determine the scope of the project, terms of reference and a working plan.

(Example: A regional task force was formed that comprised home care nurses, family physicians and specialists involved in the care of patients with leg ulcers [i.e., hematologist, dermatologist]).

2. Determine criteria for selection and select a topic using criteria.

Criteria can include prevalence of disease; evidence of underuse, overuse or misuse of interventions and existence of an evidence-based guideline. (Example: A review was performed of the extent of community-based care of chronic wounds. The most prevalent type of wound was identified as venous leg ulcer. The existence of high-level evidence for management was confirmed.)

3. Check if adaptation is feasible.

Determine if a guideline is available. (Example: A systematic search was performed. Numerous high-quality guidelines available from credible agencies [RCN, SIGN, RNAO] were identified.)

4. Identify necessary resources and skills.

Resources to consider include a high level of commitment by members of the panel, funds to cover costs of meetings, and qualified people to manage the project. Necessary skills include expertise in content, critical appraisal, retrieval of information, implementation and policy. (Example: A need for input from family physicians, nurses and specialists was identified. Methodological support for the panel by local researchers was negotiated.)

5. Complete tasks of the set-up phase.

Tasks include development of terms of reference, declaration of competing interests and process for consensus, identification of endorsement bodies, determination of authorship of the guideline and development of strategies for dissemination and implementation. (Example: Members of the group decided to function as a working group and share authorship, providing reports to the home care authority and nursing agencies involved.)

6. Write the plan for adaptation.

The plan may include details of the topic, membership of the panel, declaration of competing interests and a proposed timeline. (Example: A plan and a timeline for completion were agreed upon by the working group.)

PHASE II - Adaptation

Module: Scope and purpose

7. Determine and clarify the health-related question.

Focus on the Population of interest, the Intervention of interest, the Professions to which the guideline is to be targeted and the Outcomes and Health care setting of interest (PIPOH). (Example: Using the PIPOH approach, the group decided that the population of interest was people with venous leg ulcers; the interventions of interest were assessment and management of these patients; the professions targeted were community nurses; the outcome of interest was healed ulcers; and the health care setting was the community.)

Module: Search and screen

8. Search for guidelines and other relevant documentation.

Search for relevant guidelines, systematic reviews and reviews of health-related technology that have been published since the guideline. (Example: The group of researchers searched for relevant guidelines.)

9. Screen the retrieved guidelines and record their characteristics and content. Perform a preliminary screening to determine if the guidelines retrieved are relevant to the topic. (Example: The working group identified 8 relevant guidelines.)

10. Eliminate a large number of the retrieved guidelines using the AGREE instrument. Use the rigour dimension of the AGREE tool to assess the quality of the guideline. Include for further assessment only those that are of highest quality.

Module: Assess guidelines

11. Assess the quality of the guideline.

Use the AGREE instrument to assess quality. We suggest that 2 to 4 members do this step independently.

12. Assess the currency of the guideline.

Review the search and dates of publication of the guideline to ascertain inclusion of the most current evidence. This step requires input from an information scientist and content experts. (Example: Four guidelines for management of leg ulcers were eliminated after assessment of rigour and currency.) 
Box 1: Phases, modules and steps in the adaptation of a guideline using the ADAPTE process. A scenario of care of patients with leg ulcers is included as an example. (Part 2 of 2)

13. Assess the content of the guideline.

Content can be considered in either of 2 formats: recommendations provided and grouped by guideline or recommendations grouped by similarity (e.g. topic covered). (Example: Assessment of a leg wound was compared and in all of the high-quality guidelines, an ABPI was taken along with a detailed clinical assessment.)

14. Assess the consistency of the guideline.

Assess the search strategy and selection of evidence supporting the recommendations, the consistency between selected evidence and how developers summarize and interpret the evidence, and the consistency between the interpretation of the evidence and the recommendations. (Example: The ABPI was supported with high-level evidence in 4 guidelines.)

15. Assess the acceptability and applicability of the recommendations.

Module: Decision and selection

16. Review assessments.

Provide the panel with all documents summarizing the review, including the AGREE results and recommendations. (Example: The AGREE scores were displayed on bar graphs to easily differentiate quality scores. The recommendations for assessment and management were synthesized on a matrix of recommendations including the levels of evidence.)

17. Select among guidelines and recommendations to create an adapted guideline. Consider the following options: reject the whole guideline; accept the whole guideline, including summary of evidence and recommendations; accept the summary of evidence; accept specific recommendations; modify specific recommendations. (Example: One main guideline was used because the recommendations compared well with the other high-quality guidelines and the practice-based recommendations were well-stated.)

Module: Customization

18. Prepare a draft of the adapted guideline.

Prepare a draft document respecting the needs of the end-users and providing a detailed explanation of the process used to derive the recommendations. (Example: The co-chair of the working group compiled the results of the deliberations and wrote the local protocol.)

PHASE III - Finalization

Module: External review and acknowledgement

19. Seek feedback on the draft adapted guideline from those who would be using it. Include targeted users of the guideline, such as clinicians, managers and policy-makers. Ask whether they agree with recommendations, whether gaps exist, whether the guideline is acceptable and whether it has any resource-related implications. (Example: A copy was circulated for comment to all family physicians and home care nurses in the region.)

20. Consult with endorsement bodies.

Engage relevant professional organizations and societies to endorse the guidelines. (Example: Home care authority and nursing agencies endorsed the protocol as "usual care" for referrals for care of leg ulcers.)

21. Consult with developers of guidelines used as sources.

Send the adapted guideline to the developers, especially if changes were made to the recommendations. (Example: No substantive changes were made to recommendations so this step was not undertaken.)

22. Acknowledge source documents.

Cite references for all source documents in the final document and ensure that any necessary copyright-related permissions are obtained. (Example: The key guidelines used for the local protocol were cited.)

Module: After-care planning

23. Plan for aftercare of the adapted guideline.

Decide on a date for review and a plan for a repeat search and modification. (Example: An update 3 years after the initial local protocol was undertaken but no new evidence was found to change the recommendations.)

Module: Final production

24. Produce a final document of the guideline.

Include details on tools for implementation, including information for patients. The final document should be easily accessible to end-users. (Example: A formal, guideline-style protocol was written. Additionally, a 1-page algorithm was developed for community nurses and physicians to use.)

Note: RCN = Royal College of Nursing; SIGN = Scottish Intercollegiate Guidelines Network; RNAO = Registered Nurses Association of Ontario; PIPOH = Population, Intervention, Professions, Outcomes, and Health care setting; AGREE = Appraisal of Guidelines Research and Evaluation; $A B P I=$ ankle-brachial pressure index. 
lines Framework for Improvement. ${ }^{27}$ They based their classification on interviews with focus groups of Norwegian general practitioners about factors affecting adherence to clinical practice guidelines for ordering diagnostic images for back pain. Newly identified barriers were lack of expectancy that adherence to guidelines will lead to the desired process of health care, emotional difficulty with adherence, improper access to actual or alternative health care services and pressure to do otherwise from health care providers and organizations. ${ }^{27}$

More recently, the Clinical Practice Guidelines Framework for Improvement was expanded. In a study targeting the identification of barriers to and facilitators of implementing shared decision-making in clinical practice, each type of barrier was provided with a specific definition. ${ }^{28}$ The intention was to standardize the reporting of barriers to and facilitators of use of knowledge in the context of health care..$^{28}$ Barriers were defined as factors that would limit or restrict implementation of shared decision-making in clinical practice. ${ }^{29}$ More importantly, the Clinical Practice Guidelines Framework for Improvement was transferred into a list of potential facilitators of use of knowledge in clinical practice. ${ }^{28}$ Facilitators were defined as factors that would promote or help implement shared decision-making in clinical practice.

Sometimes we forget that the same factor may sometimes be identified both as a barrier to and as a facilitator of use of knowledge, showing the importance of developing a more comprehensive and integrated understanding of both barriers and facilitators concurrently. ${ }^{30,31}$ The Clinical Practice Guidelines Framework for Improvement was further extended to include the attributes of innovation as proposed by the Diffusion of Innovation theory. ${ }^{32}$ As a result, except for the barrier known as lack of awareness (i.e., not knowing of the existence of a guideline) and the facilitator known as awareness (i.e., knowing about a guideline), the other factors initially proposed by the Clinical Practice Guidelines Framework for Improvement were potential barriers or facilitators. One new barrier, "forgetting" (i.e., inadvertently omitting to attend to something) was also identified.

This revised version of the Clinical Practice Guidelines Framework for Improvement was used in a systematic review of barriers to and facilitators of implementing shared decision-making in clinical practice. ${ }^{33}$ The framework was applied successfully in extracting data from 41 publications covering 38 unique studies. ${ }^{33}$ The corresponding definitions of each of the potential barriers to and facilitators of use of knowledge in the health care context are provided in Appendix 1 (available at www.cmaj.ca/cgi/content/full/cmaj.081232/DC1).

\section{Tools for assessment}

To clearly identify barriers to and facilitators of use of knowledge in health care practices, assessment of them in a valid and reliable fashion is needed. Considerable interest exists in the idea of instruments for valid and reliable assessment of barriers to and facilitators of use of knowledge that can be used by various end-users who are trying to implement knowledge. Based on the Clinical Practice Guidelines Framework for Improvement, a tool to assess barriers to adherence to guidelines for hand-specific hygiene was developed and tested on a group of 21 clinicians of infectious disease. ${ }^{34}$ The tool uses a six-point Likert scale and has two sections: attitudinal statements about practice guidelines in general and specific statements regarding hand hygiene. The survey was administered twice, at two-week intervals. The tool known as Attitudes Regarding Practice Guidelines was found to have good reliability. ${ }^{34}$ However, the authors concluded that their tool needed to undergo further testing and adaptation as a general measure of potential barriers to adherence to practice guidelines. ${ }^{34}$

One of us (FL) completes regular audits of practice as part of a primary health care group. In a large, urban site for teaching family medicine (i.e., with 20 clinical teachers and 24 residents in family medicine as well as three nurses), the residents recently completed an audit of ambulatory care of patients with type 2 diabetes mellitus. One of the clinical teachers supervised a group of four residents in the completion of this audit. Residents reviewed the relevant practice guidelines and assessed their quality using the AGREE checklist. Based on this appraisal of quality, they retained the recommendations of the guidelines of the Canadian Diabetes Association. Based on these recommendations, they created a grid for extracting data on whether the recommendations were implemented. Forty patient charts were chosen randomly and the percentage of cases that followed the recommendations of guidelines was calculated.

The results of the audit were presented to the health care team for discussion. For example, results showed that a test for glycosylated hemoglobin was performed every three months, as recommended by the guidelines of the Canadian Diabetes Association (i.e., level D, consensus) in only 30\% of cases. Based on the Clinical Practice Guidelines Framework for Improvement, discussion by the group revealed that barriers perceived by health care providers included lack of agreement with the recommendation because it was too rigid or artificial, factors associated with environment such as not having enough staff to carry out the recommendation, lack of agreement with the applicability of this recommendation to the population served by the practice based on the characteristics of the patient because some patients had very stable results in the past, and external factors, such as perceived inability to reconcile patient preferences with compliance to this recommendation.

This scenario shows that groups of providers in real clinical settings can take advantage of this checklist to identify barriers to carrying out recommendations in guidelines for practice. Once the barriers are made explicit, solutions can be expected to be brought forward for the benefit of patients. Without ignoring that one solution usually does not fit all, this checklist has the potential to help groups of providers reconcile their diverse perspectives because it is evidence-based, extensive and explicit.

\section{Gaps in the implementation of evidence}

Although many current research-based initiatives focus on implementing guidelines and assessing factors influencing use of knowledge in health care practices, many challenges remain that will need to be addressed by rigorous research. First, evalu- 
ation of the ADAPTE process is needed to determine its impact on the implementation of guidelines. Second, validated methods are needed to assess barriers to and facilitators of the translation of research into clinical practice..$^{29,35,36}$ Researchers and clinicians may want to consider using existing models that have been tested, such as the Clinical Practice Guidelines Framework for Improvement (i.e., in its latest version), to conduct studies on barriers to and facilitators of assessment. ${ }^{25}$ Lastly, more will need to be done to reconcile the recommendations of practice guidelines to the sharing of care-related decisions with patients - the core concept of patient-centred care.

This article has been peer reviewed.

Competing interests: None declared.

Contributors: Margaret Harrison, Béatrice Fervers and Ian Graham, who are founding members of the ADAPTE group, were involved in the development of the methodology of the manuscript, and conceptualized and drafted the section on adaptation of guidelines. France Légaré and Ian Graham were involved in the conceptualization and drafting of the section on assessment of barriers. All of the authors critically revised the manuscript and approved the final version submitted for publication.

Funding: No external funding was received for this paper.

\section{REFERENCES}

1. Graham ID, Logan J, Harrison MB, et al. Lost in knowledge translation: time for a map? J Contin Educ Health Prof 2006;26:13-24.

2. Straus S, Haynes RB. Managing evidence-based knowledge: the need for reliable, relevant and readable resources. CMAJ 2009;180:942-5.

3. Straus SE, Richardson WS, Glasziou P, et al. Evidence based medicine: how to practice and teach it. Edinburgh (UK): Elsevier; 2005. p. 95-7.

4. Burgers JS, Grol R, Zaat JO, et al. Characteristics of effective clinical guidelines for general practice. Br J Gen Pract 2003;53:15-9.

5. National Institute for Health and Clinical Excellence. Available: www.nice.org.uk (accessed 2009 Sept. 15).

6. Grimshaw JM, Thomas RE, MacLennan G, et al. Effectiveness and efficiency of guideline dissemination and implementation strategies. Health Technol Assess 2004;8:iii-iv, 1-72

7. Toman C, Harrison MB, Logan J. Clinical practice guidelines: necessary but not sufficient for evidence-based patient education and counseling. Patient Educ Couns 2001;42:279-87.

8. GRADE Working Group. Grading quality of evidence and strength of recommen dations. BMJ 2004;328:1490-7.

9. AGREE Collaboration. Development and validation of an international appraisal instrument for assessing the quality of clinical practice guidelines: the AGREE Project. Qual Saf Health Care 2003;12:18-23.

10. Harrison MB, Graham ID, Lorimer K, et al. Leg-ulcer care in the community, before and after implementation of an evidence-based service. CMAJ 2005;172:1447-52.

11. Ray-Coquard I, Philip T, Lehmann M, et al. Impact of a clinical guidelines program for breast and colon cancer in a French cancer centre. JAMA 1997;278 1591-5.

12. Ray-Coquard I, Philip T, de Laroche G, et al. A controlled "before-after" study: impact of a clinical guidelines programme and regional cancer network organization on medical practice. Br J Cancer 2002;86:313-21.

13. Harrison MB, Graham ID, Lorimer K, et al. Nurse clinic versus home delivery of evidence-based community leg ulcer care: a randomized health services trial. BMC Health Serv Res 2008;8:243.

14. Fretheim A, Schunemann HJ, Oxman AD. Improving the use of research evidence in guideline development: 3. Group composition and consultation process. Health Res Policy Syst 2006;4:15.

15. Graham ID, Harrison MB, Brouwers M, et al. Facilitating the use of evidence in practice: evaluating and adapting clinical practice guidelines for local use by health care organizations. J Obstet Gynecol Neonatal Nurs 2002;31:599-611.

16. Graham ID, Harrison MB, Brouwers M. Evaluating and adapting practice guidelines for local use: a conceptual framework. In: Pickering S, Thompson J, editors. Clinical governance in practice. London (UK): Harcourt; 2003. p. 213-29.

17. Graham ID, Harrison MB, Lorimer K, et al. Adapting national and international leg ulcer practice guidelines for local use: the Ontario Leg Ulcer Community Care Protocol. Adv Skin Wound Care 2005;18:307-18.

18. Burgers JS, Cluzeau FA, Hanna SE, et al. Characteristics of high-quality guidelines: evaluation of 86 clinical guidelines developed in ten European countries and Canada. Int J Technol Assess Health Care 2003;19:148-57.

19. Fervers B, Burgers JS, Haugh M, et al. Adaptation of clinical guidelines: literature review and proposition for a framework and procedure. Int J Qual Health Care 2006; 18:167-76

20. Shiffman RN, Shekelle P, Overhage JM, et al. Standardized reporting of clinical practice guidelines: a proposal from the Conference on Guideline Standardization. Ann Intern Med 2003;139:493-8.

21. Verkerk K, Van Veenendaal H, Severens JL, et al. Considered judgement in evidence-based guideline development. Int J Qual Health Care 2006;18:365-9.

22. Grol R, Dalhuijsen J, Thomas S, et al. Attributes of clinical guidelines that influence use of guidelines in general practice: observational study. BMJ 1998;317:858-61.

23. AGREE Collaboration. International assessment of quality clinical practice guidelines in oncology using the Appraisal of Guidelines and Research and Evaluation instrument. J Clin Oncol 2004;22:2000-7.

24. Eccles M, Grimshaw J, Walker A, et al. Changing the behavior of healthcare professionals: the use of theory in promoting the uptake of research findings. J Clin Epidemiol 2005;58:107-12.

25. Cabana MD, Rand CS, Powe NR, et al. Why don't physicians follow clinical practice guidelines? A framework for improvement. JAMA 1999;282:1458-65.

26. Ajzen I. Attitudes, personality and behavior: Berkshire (UK): Open University Press; 1988.

27. Espeland A, Baerheim A. Factors affecting general practitioners' decisions about plain radiography for back pain: implications for classification of guideline barriers - a qualitative study. BMC Health Serv Res 2003;3:8.

28. Légaré F, O'Connor AM, Graham ID, et al. Primary health care professionals views on barriers and facilitators to the implementation of the Ottawa Decision Support Framework in practice. Patient Educ Couns 2006;63:380-90.

29. Cabana MD, Rand CS, Powe NR, et al. Why don't physicians follow clinical practice guidelines? A framework for improvement. JAMA 1999;282:1458-65

30. Graham ID, Logan J, O'Connor A, et al. A qualitative study of physicians' perceptions of three decision aids. Patient Educ Couns 2003;50:279-83.

31. Kennedy T, Regehr G, Rosenfield J, et al. Exploring the gap between knowledge and behavior: a qualitative study of clinician action following an educational intervention. Acad Med 2004;79:386-93.

32. Rogers EM. Diffusion of innovations. 4th ed. New York (NY): The Free Press; 1995.

33. Légaré F, Ratte S, Gravel K, et al. Barriers and facilitators to implementing shared decision-making in clinical practice: update of a systematic review of health professionals' perceptions. Patient Educ Couns 2008;73:526-35.

34. Larson E. A tool to assess barriers to adherence to hand hygiene guideline. Am J Infect Control 2004;32:48-51.

35. Davis DA, Taylor-Vaisey A. Translating guidelines into practice. A systematic review of theoretic concepts, practical experience and research evidence in the adoption of clinical practice guidelines. CMAJ 1997;157:408-16.

36. Saillour-Glenisson F, Michel P. Individual and collective facilitators and barriers to the use of clinical guidelines by physicians: a literature review [article in French]. Revue Épidémiologique de Santé Publique. 2003;51:65-80.

Correspondence to: Dr. Margaret B. Harrison, School of Nursing, Queen's University, 78 Barrie St., Kingston ON K7L 3N6; margaret.b.harrison@queensu.ca

\section{ADAPTE Group}

Melissa Brouwers, George Browman, Jako Burgers, Bernard Burnand, Margaret B. Harrison, Béatrice Fervers, Ian Graham, Jean Latreille, Najoua Mlika-Cabanne, Louise Paquet, Raghu Rajan, Magali Remy-Stockinger, Anita Simon, Joan Vlayen and Louise Zitzelsberger

The book Knowledge Translation in Health Care: Moving from Evidence to Practice, edited by Sharon Straus, Jacqueline Tetroe and Ian D. Graham and published by Wiley-Blackwell in 2009 , includes the topics addressed in this series.

\section{Articles to date in this series}

- Straus SE, Tetroe J, Graham I. Defining knowledge translation. CMAJ 2009;181:165-8.

- Brouwers M, Stacey D, O'Connor A. Knowledge creation: synthesis, tools and products. CMAJ 2009.DOI:10.1503 /cmaj.081230

- Kitson A, Straus SE. The knowledge-to-action cycle: identifying the gaps. CMAJ 2009.DOI:10.1503/cmaj.081231 